\title{
Elevated Peripheral Neutrophils and Matrix Metalloproteinase 9 as Biomarkers of Functional Outcome Following Subarachnoid Hemorrhage
}

\author{
S. H.-Y. Chou • S. K. Feske • S. L. Simmons • R. G. J. Konigsberg • S. C. Orzell • \\ A. Marckmann • G. Bourget • D. J. Bauer • P. L. De Jager • R. Du • K. Arai • E. H. Lo • \\ M. M. Ning
}

Received: 9 September 2011 /Revised: 10 October 2011 /Accepted: 12 October 2011 /Published online: 10 November 2011

(C) Springer Science+Business Media, LLC 2011

\begin{abstract}
There is growing evidence supporting the role of inflammation in early brain injury and cerebral vasospasm following subarachnoid hemorrhage (SAH). Matrix metalloproteinases (MMPs) are released by inflammatory cells and can mediate early brain injury via disruption of the extracellular matrix and mediate vasospasm by cleaving endothelin-1 into vasoactive fragments. We hypothesize that inflammation marked by neutrophil elevation and MMP-9 release in human SAH is associated with vasospasm and with poor clinical outcome. We enrolled consecutive SAH subjects $(N=55)$, banked serial blood and cerebrospinal fluid (CSF) samples, and evaluated their 3-month modified Rankin scores (mRS). Vasospasm was defined as $>50 \%$ vessel caliber reduction on angiography 6-8 days post-SAH. A poor outcome was defined as mRS $>2$. We compared blood leukocyte and neutrophil counts during
\end{abstract}

Eng H. Lo and MingMing Ning contributed equally to this manuscript.

S. H.-Y. Chou • S. K. Feske • P. L. De Jager

Department of Neurology, Brigham and Women's Hospital, Harvard Medical School,

Boston, MA, USA

S. L. Simmons · R. G. J. Konigsberg • S. C. Orzell • D. J. Bauer Department of Neurology, Brigham and Women's Hospital, Boston, MA, USA

\section{A. Marckmann}

Department of Neurology, Massachusetts General Hospital, Boston, MA, USA

M. M. Ning

Department of Neurology, Massachusetts General Hospital, Harvard Medical School,

Boston, MA, USA
post-SAH days 0-14 with respect to vasospasm and 3-month outcome. In a subset of SAH subjects $(N=35)$, we compared blood and CSF MMP-9 by enzyme-linked immunosorbent assay (ELISA) on post-SAH days $0-1,2-3,4-5,6-8$, and 10-14 with respect to vasospasm and to 3 -month outcome. Persistent elevation of blood leukocyte $(p=0.0003)$ and neutrophil $(p=0.0002)$ counts during post-SAH days $0-14$ are independently associated with vasospasm after adjustment for major confounders. In the same time period, blood neutrophil count (post-SAH days $2-3, p=0.018$ ), blood MMP-9 (post-SAH days $4-5, p=0.045$ ), and CSF MMP-9 (post-SAH days $2-3, p=0.05$ ) are associated with poor 3month SAH clinical outcome. Neutrophil count correlates with blood MMP-9 (post-SAH days $6-8, R=0.39 ; p=0.055$; post-SAH days $10-14, R=0.79 ; p<0.0001$ ), and blood MMP-9 correlates with CSF MMP-9 (post-SAH days 4-5,

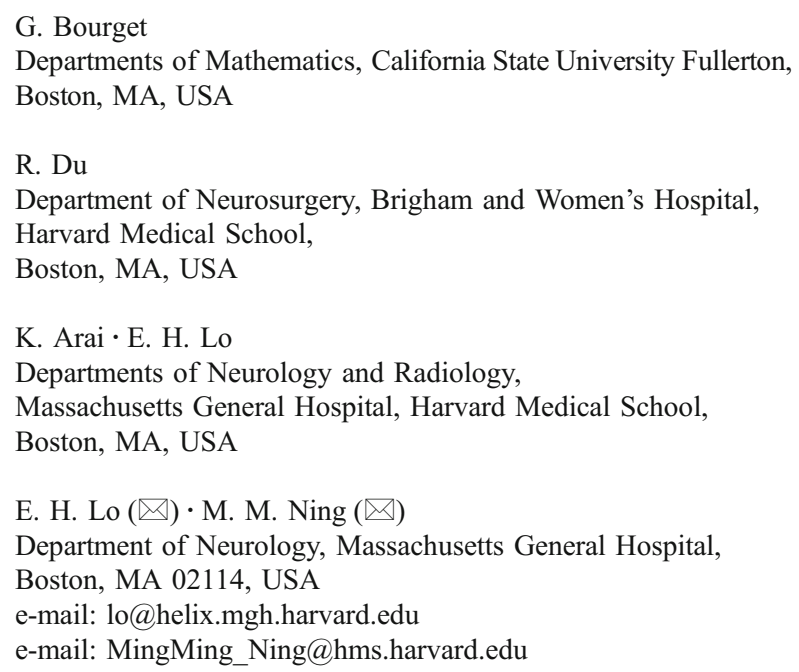


$R=0.72 ; p=0.0002)$. Elevation of CSF MMP-9 during postSAH days $0-14$ is associated with poor 3-month outcome ( $p$ $=0.0078)$. Neither CSF nor blood MMP-9 correlates with vasospasm. Early rise in blood neutrophil count and blood and CSF MMP-9 are associated with poor 3-month SAH clinical outcome. In blood, neutrophil count correlates with MMP-9 levels, suggesting that neutrophils may be an important source of blood MMP-9 early in SAH. Similarly, CSF and blood MMP-9 correlate positively early in the course of SAH, suggesting that blood may be an important source of CSF MMP-9. Blood and CSF MMP-9 are associated with clinical outcome but not with vasospasm, suggesting that MMP-9 may mediate brain injury independent of vasospasm in SAH. Future in vitro studies are needed to investigate the role of MMP-9 in SAH-related brain injury. Larger clinical studies are needed to validate blood and CSF MMP-9 as potential biomarkers for SAH outcome.

Keywords Subarachnoid hemorrhage $\cdot$ Biomarker $\cdot$ Matrix metalloproteinase $\cdot$ Inflammation · Neutrophils ·

Vasospasm · Outcome

\section{Introduction}

Spontaneous subarachnoid hemorrhage (SAH) is one of the leading causes of neurologic morbidity and mortality, accounting for $27 \%$ of all stroke-related years of potential life lost before age 65 [1]. SAH is associated with early brain injury, which is the primary cause of mortality in SAH patients and may be associated with the pathogenesis of cerebral vasospasm [2]. Cerebral vasospasm is a known complication of $\mathrm{SAH}$ and another important cause of morbidity and mortality in SAH patients who survive aneurysm treatment [3]. How early brain injury and cerebral vasospasm ultimately affect long-term SAH outcome in human subjects remain incompletely understood [4], and there are few clinical molecular biomarkers for vasospasm and SAH outcome.

There is growing evidence that inflammation plays a key role in early brain injury and cerebral vasospasm [2, 5, 6]. Elevation of leukocyte adhesion molecules and cytokines in cerebrospinal fluid (CSF) and serum are associated with changes in cerebral blood flow in SAH [7, 8]. Multiple animal and cross-sectional human studies have reported association between leukocytosis and the development of vasospasm or ischemia [9-11] and poor outcome [12-15] following $\mathrm{SAH}$. The mechanism via which inflammation causes narrowing of cerebral arteries and brain injury is incompletely understood. Inflammatory cells such as leukocytes are a major source of MMPs after brain injury [16]. MMP-9, in particular, may mediate early brain injury in SAH by causing neuronal apoptosis through disruption of neuron-extracellular matrix interaction [17, 18]. MMPs are also implicated in the disruption of blood-brain barrier (BBB) integrity, progression of cerebral edema, and worsening cerebral ischemic injury [19], all of which may affect SAH clinical outcome. Furthermore, MMPs 2 and 9 can cleave big endothelin-1 (ET-1) into strongly vasoactive segments which further promote neutrophilic MMP release, creating a positive feedback loop that potentially mediates cerebral vasospasm [20-22]. MMP-9 therefore may mediate a poor SAH outcome via different and divergent pathogenic mechanisms and may be the link between inflammation and the genesis of "spasmogens" such as ET-1 in cerebral vasospasm following SAH. We hypothesize inflammation marked by leukocyte and neutrophil elevation in SAH is associated with MMP-9 elevation in blood and CSF, leading to vasospasm and worse $\mathrm{SAH}$ clinical outcome, and that peripheral leukocyte and neutrophil counts and blood and CSF MMP-9 may be useful biomarkers for vasospasm and outcome following SAH.

\section{Methods}

\section{Subject Selection}

Fifty five consecutive SAH patients within $96 \mathrm{~h}$ of spontaneous SAH were prospectively enrolled, and their blood and available CSF were sampled on post-SAH days 0-1, 2-3, 4-5, 6-8, and 10-14. CSF samples were acquired only from patients who had an external ventricular drain (EVD) placed for clinical indications. Patients with traumatic SAH, pregnancy, end-stage renal or hepatic disease, intracranial malignancies, or infectious meningitis were excluded. We selected a subcohort of subjects with both blood and CSF samples available for MMP-9 analysis $(N=35)$. All subjects were consented in accordance with institutional review board approved protocols.

Daily peripheral leukocyte and neutrophil count is extracted from subjects' medical records. Per clinical protocol, all SAH patients underwent daily complete blood count measurement using automatic flow cytometry (ADVIA 120, Siemens) by CLIO-certified clinical laboratory. For subjects who had more than one measurement on a single day, the highest measurement was recorded.

Blood samples for MMP-9 analysis were collected in heparincoated tubes and immediately centrifuged at 3,900 rpm for $15 \mathrm{~min}$, aliquotted, and then stored frozen at $-80^{\circ} \mathrm{C}$ until analysis. CSF samples were collected under sterile conditions over $30 \mathrm{~min}$ from the anti-reflux burette in the EVD system. Upon collection, all samples were immediately centrifuged (3,900 rpm, $15 \mathrm{~min}$ at room temperature) and separated from cellular debris, aliquoted, and stored frozen at $-80^{\circ} \mathrm{C}$.

Total MMP-9 (active plus pro-MMP-9; R\&D Systems, Minneapolis, MN) were measured using solid-phase ELISA by blinded investigators. All assays were run in duplicates 
using internal controls for each batch. Gel zymography was performed to assess MMP-9 activity and isoforms via previously described methods [23].

Angiographic vasospasm was defined as $>50 \%$ reduction in diameter of any vessel on angiography on post-SAH days 6-8. Per institutional protocol, all SAH patients underwent digital subtraction cerebral angiography (DSCA) upon initial presentation and then again on post-SAH days 6-8 for evaluation of angiographic vasospasm. Severity of vasospasm was determined by comparing subsequent to baseline DSCA. The total number of vessel segments with vasospastic changes did not affect the grading of vasospasm. When multiple vessels were involved, subjects were classified based on the most severely affected vessel. Subjects who had several subsequent DSCAs were classified according to the most severe vasospasm seen among all DSCA.

Long-term functional outcome at 3-month post-SAH were prospectively assessed using modified Rankin score (mRS) via telephone interview. A standardized questionnaire was used to perform $\mathrm{mRS}$ scoring to reduce inter-rater variability. A poor outcome was defined as $\mathrm{mRS}>2$.

\section{Statistical Analysis}

Continuous variables were compared using two-tailed Student's $t$-test or Wilcoxon rank sum test depending on data normality. Depending on data normality, Pearson or Spearman correlation was used to measure strength of association between continuous variables. Categorical variables were compared using Chi-square test. We used longitudinal regression analysis to model data with repeated measurements. All statistical analyses were performed using JMP 8.0 and SAS 9.2.

\section{Results}

\section{Baseline Characteristics}

Subject characteristics of the entire SAH cohort $(n=55)$ are summarized in Table 1. Mean age, gender distribution, aneurysm locations, and incidences of vasospasm and poor outcome are comparable between the large SAH cohorts and the subset of subjects selected for MMP-9 analysis. Of the overall cohort, $49 \%$ presented with Hunt and Hess (HH) grade of 3 and above, whereas $60 \%$ of the MMP-9 subcohort presented with $\mathrm{HH}$ grade of 3 and above. The large majority of subjects have Fisher grade $3 \mathrm{SAH}$.

Elevated Blood Leukocyte and Neutrophil Counts are Associated with Vasospasm

Subjects who developed angiographic vasospasm have higher leukocyte counts throughout post-SAH days $0-14$ ( $p=0.0003$;
Table 1 Baseline characteristics of study cohort

\begin{tabular}{|c|c|}
\hline Characteristics & $(\mathrm{N}=55)$ \\
\hline Age (mean years) & $53(+/-16)$ \\
\hline Female Gender (\%) & $31(56 \%)$ \\
\hline \multicolumn{2}{|l|}{ Hunt and Hess Grade } \\
\hline 1 & $7(13 \%)$ \\
\hline 2 & $21(38 \%)$ \\
\hline 3 & $13(24 \%)$ \\
\hline 4 & $9(16 \%)$ \\
\hline 5 & $5(9 \%)$ \\
\hline \multicolumn{2}{|l|}{ Fisher Grade } \\
\hline 1 & $3(5 \%)$ \\
\hline 2 & $11(20 \%)$ \\
\hline 3 & $38(69 \%)$ \\
\hline 4 & $3(5 \%)$ \\
\hline \multicolumn{2}{|l|}{ Aneurysm Location } \\
\hline Acom & $21(38 \%)$ \\
\hline Pcom & $11(20 \%)$ \\
\hline Angio Negative & $10(18 \%)$ \\
\hline ICA & $3(5 \%)$ \\
\hline PICA & $4(7 \%)$ \\
\hline MCA & $3(5 \%)$ \\
\hline Basilar & $1(2 \%)$ \\
\hline ACA & $2(4 \%)$ \\
\hline Angiographic Vasospasm & $22(60 \%)$ \\
\hline \multicolumn{2}{|l|}{ 3-month Outcome } \\
\hline $\mathrm{mRS}>2(\mathrm{bad})$ & $15(28 \%)$ \\
\hline $\mathrm{mRS} \leq 2($ good $)$ & $39(72 \%)$ \\
\hline \multicolumn{2}{|l|}{ 6-month Outcome } \\
\hline $\mathrm{mRS}>2(\mathrm{bad})$ & $10(20 \%)$ \\
\hline mRS $\leq 2$ (good) & $41(80 \%)$ \\
\hline
\end{tabular}

Fig. 1). Individually, elevated peripheral leukocyte counts on post-SAH days $2(p=0.037), 3(p=0.046), 4(p=0.028), 5$ $(p=0.025), 6(p=0.036), 7 \quad(p=0.0018), 8 \quad(p=0.0097), 9$ $(p=0.0007), 10 \quad(p=0.0003)$, and $11 \quad(p=0.019)$ are associated with angiographic vasospasm. After adjustment for potential confounders including $\mathrm{HH}$ grade, age, and modality of SAH surgical treatment, elevated leukocyte counts throughout post-SAH days $0-14$ remain independently associated with vasospasm $(p=0.0003)$.

We also examined a specific subset of leukocytes neutrophils. Subjects who developed angiographic vasospasm have higher neutrophil counts throughout post-SAH days 0 14 ( $p=0.0027$; Fig. 2). Individually, elevated peripheral neutrophil counts on post-SAH days $0-1(p=0.018)$ and $6-$ $8(p=0.0005)$ are associated with the development of angiographic vasospasm. After adjustment for potential confounders including $\mathrm{HH}$ grade, age, and modality of SAH surgical treatment, elevated neutrophil counts throughout 
Fig. 1 Peripheral white blood cell count is elevated in SAH subjects with angiographic vasospasm (VSP $+; n=22)$ compared with those without Vasospasm (VSP-; $n=33$ ) during post-SAH days $0-14(p=0.0003)$. $*$ indicates between-group difference on that specific postSAH day is significant at a level of $p<0.05$

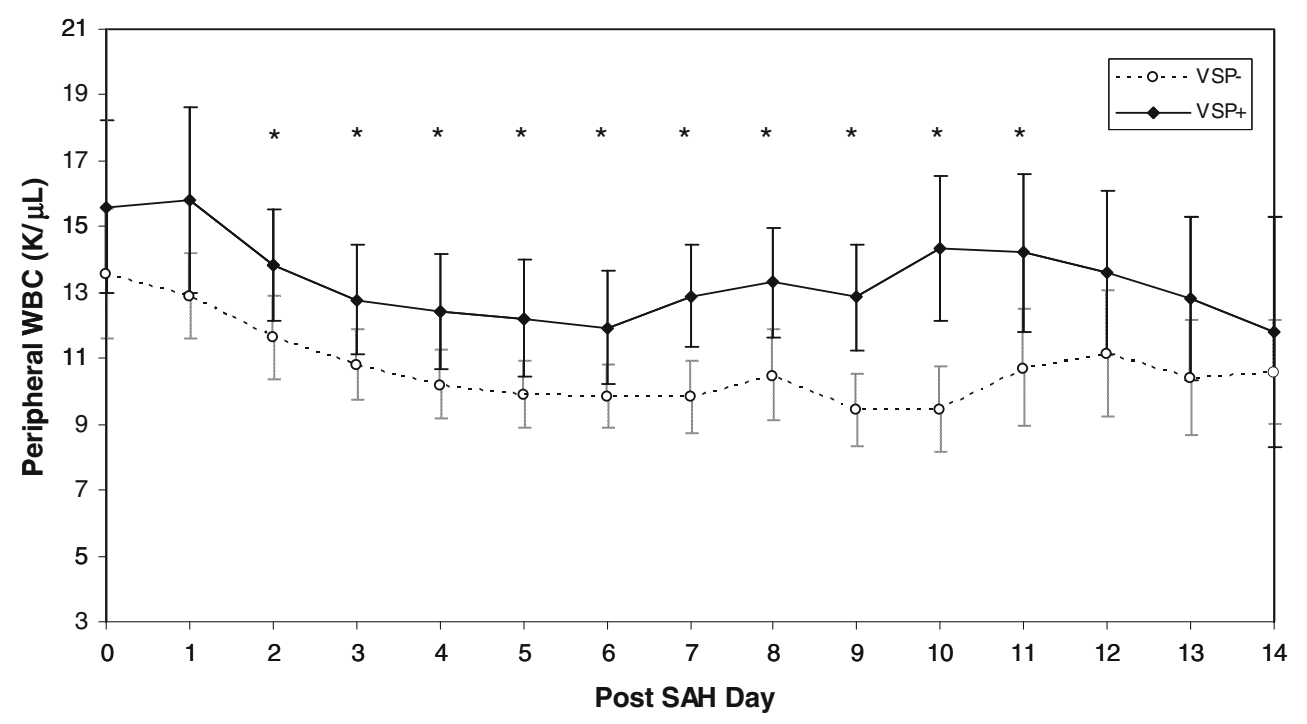

post-SAH days $0-14$ remained independently associated with vasospasm $(p=0.0002)$.

Elevated Neutrophil Count, Blood, and CSF MMP-9 are Associated with Poor 3-month SAH Outcome

Persistent blood neutrophil elevation throughout post-SAH days $0-14$ is associated with poor 3-month $\mathrm{SAH}$ outcome $(\mathrm{mRS}>2 ; p=0.018)$. Individually, elevated neutrophil count on post-SAH days $2-3$ is associated with poor 3 -month SAH outcome ( $p=0.018$; Fig. 3$)$.

Persistent blood MMP-9 elevation throughout post-SAH days $0-14$ showed a trend towards association with 3-month SAH outcome in a longitudinal regression model $(p=0.09)$. Specifically, elevated blood MMP-9 on post-SAH days $4-5$ is associated with poor 3-month SAH outcome ( $p=0.045$; Fig. 4a).

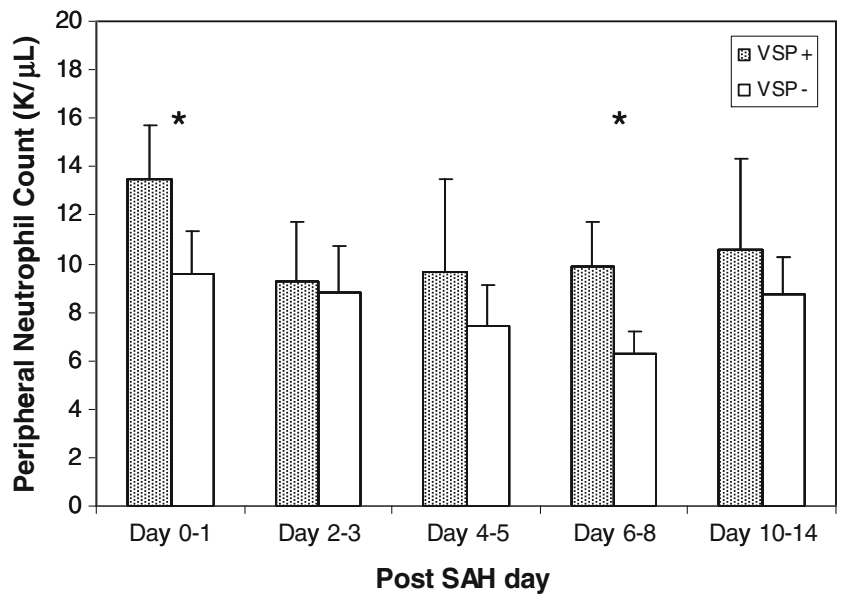

Fig. 2 Peripheral neutrophil count is elevated in SAH subjects with angiographic vasospasm (VSP+; $n=15$ ) compared with those without vasospasm (VSP-; $n=23)$ during post-SAH days $0-14(p=0.0027)$. $*$ indicates between-group difference on that specific post-SAH day is significant at a level of $p<0.05$
MMP-9 is detectable in CSF at levels approximately 200-fold lower than that in blood. Zymogram confirmed that the MMP-9 present in the CSF is enzymatically active [24], suggesting that it could functionally participate in the events triggered by SAH. Persistent elevation of CSF MMP-9 throughout post-SAH days $0-14$ is associated with poor 3-month SAH outcome $(p=0.0078)$. Specifically, elevated CSF MMP-9 on post-SAH days $2-3(p=0.05)$ is associated with poor 3-month outcome (Fig. 4b).

Neutrophil Counts, Blood MMP-9, and CSF MMP-9 Correlations

In blood, neutrophil counts correlate with MMP-9 levels on post-SAH days $6-8(R=0.39 ; p=0.055$, Fig. $5 \mathrm{a})$ and postSAH days $10-14(R=0.79 ; p<0.0001$, Fig. $5 b)$. CSF

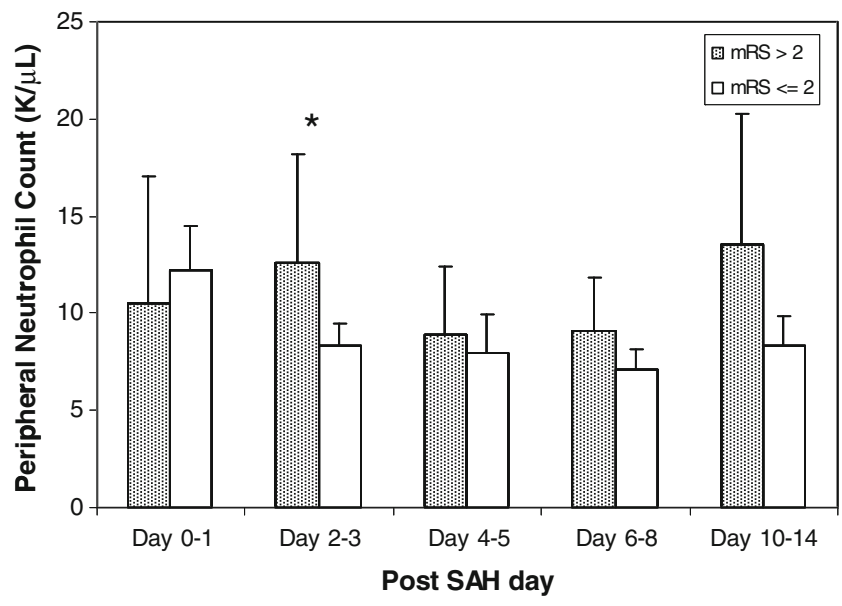

Fig. 3 Peripheral neutrophil count is elevated during post-SAH days $0-14$ in SAH subjects with poor $(\mathrm{mRS}>2 ; n=12) 3$-month outcome compared with those with good outcome ( $\mathrm{mRS} \leq 2 ; n=25 ; p=0.018)$. $*$ indicates between-group difference on that specific post-SAH day is significant at a level of $p<0.05$ 
(a)

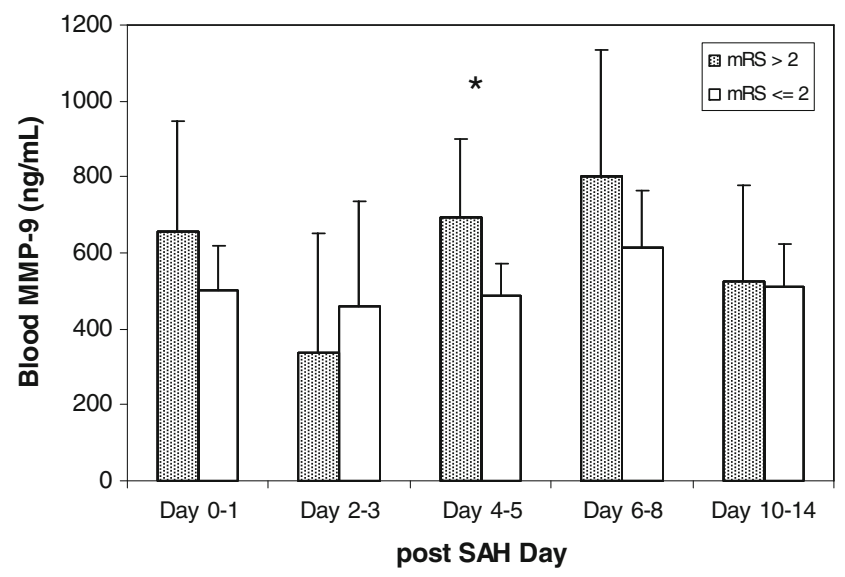

(b)

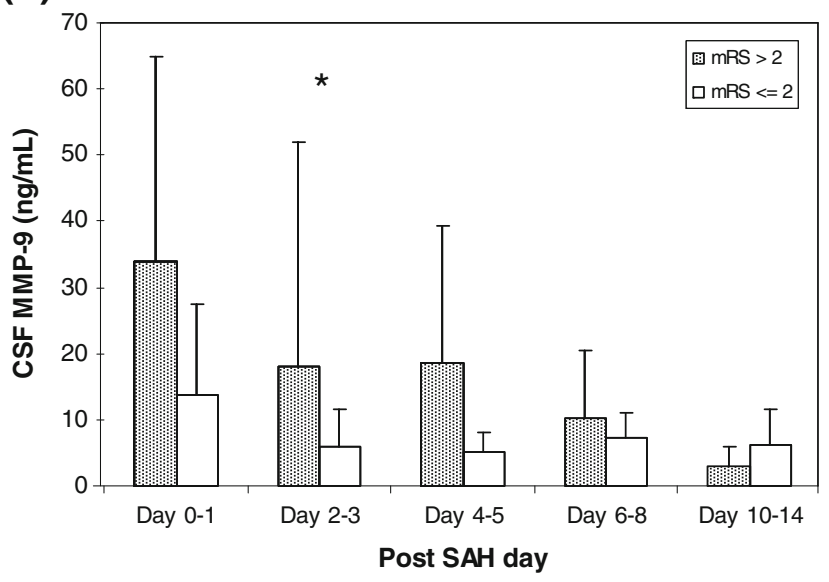

Fig. 4 a Elevation of blood MMP-9 levels during post-SAH days 014 in SAH patients showed a trend of association with poor $(\mathrm{mRS}>2$; $n=10) 3$-month outcome versus good $(\mathrm{mRS} \leq 2 ; n=21)$ outcome $(p=$ 0.09). b Elevation of CSF MMP-9 levels during post-SAH days 0-14 in SAH patients is associated with poor $(\mathrm{mRS}>2 ; n=9) 3$-month outcome versus good $(\mathrm{mRS} \leq 2 ; n=22 ; p=0.0078)$. * indicates between-group difference on that specific post-SAH day is significant at a level of $p<0.05$ )

MMP-9 correlates with blood MMP-9 on post-SAH days 4-5 $(R=0.72 ; p=0.0002$; Fig. 6). However, neither CSF $(p=0.32)$ nor blood MMP-9 $(p=0.42)$ were associated with angiographic vasospasm on statistical analysis (Fig. 7).

\section{Discussion}

We found that early elevation of blood neutrophil count is associated with both cerebral vasospasm and poor 3-month clinical outcome after SAH, and both blood and CSF MMP-9 are associated with poor 3-month SAH clinical outcome but not with vasospasm. Early blood neutrophil count is associated with blood MMP-9 levels, suggesting that neutrophils may be an important source of blood MMP-9. Similarly, early (a)

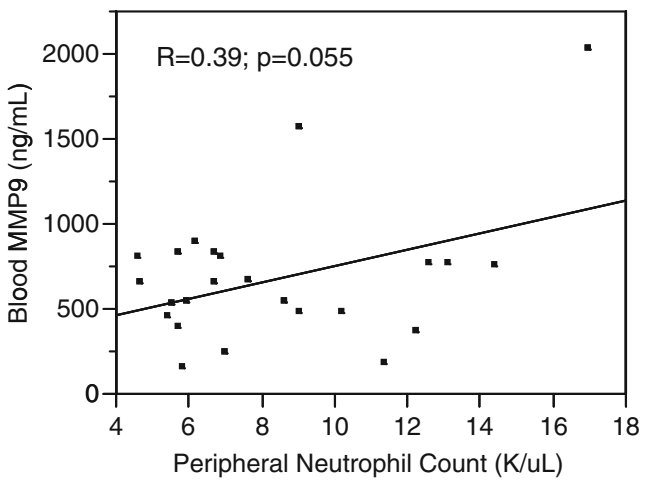

(b)

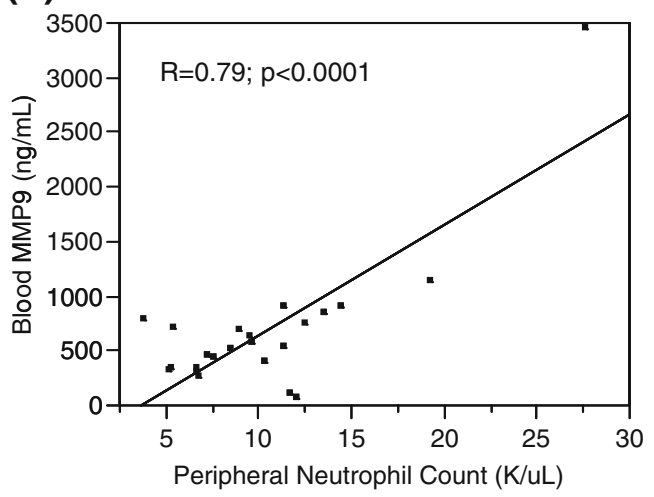

Fig. 5 Peripheral neutrophil counts correlate with blood MMP-9 levels a on post-SAH days $6-8(n=24 ; \mathrm{R}=0.39 ; p=0.055)$ and $\mathbf{b}$ on post-SAH days $10-14(n=23 ; \mathrm{R}=0.79 ; p<0.0001)$

blood MMP-9 elevation is associated with CSF MMP-9 elevation while blood MMP-9 levels are 200-fold higher than CSF MMP-9 levels, suggesting that blood MMP-9 may be an important source of CSF MMP-9 early in SAH.

Recent studies suggest MMPs may participate in the pathogenesis of cerebral vasospasm and early brain injury in multiple pathways. Activation of MMP-9 is associated with endothelial apoptosis, which plays an important role in

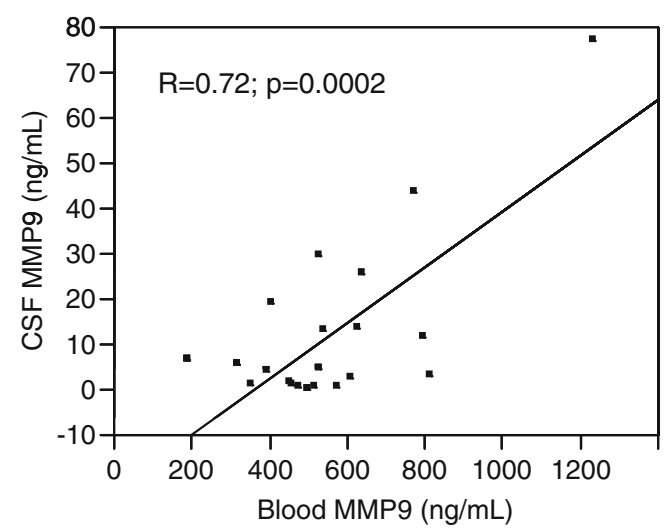

Fig. 6 Blood MMP-9 correlates with CSF MMP9 on post-SAH days 4-5 $(n=22 ; R=0.72 ; p=0.0002)$ 
(a)

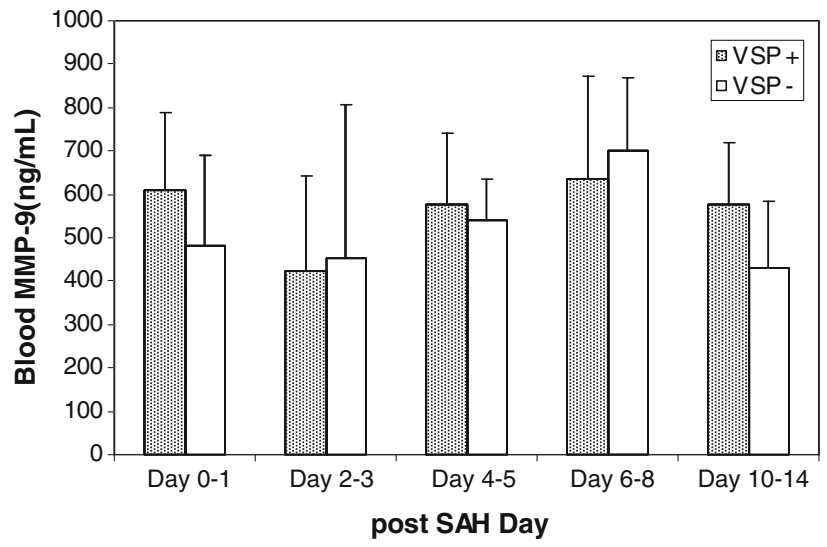

(b)

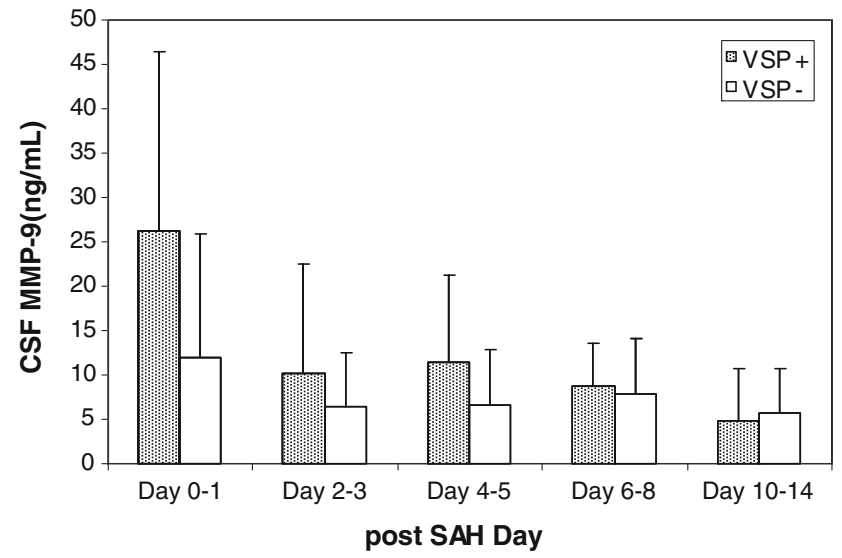

Fig. 7 a Blood MMP-9 levels during post-SAH days 0-14 in SAH patients showed no association with presence (VSP+; $n=17)$ or absence of angiographic vasospasm (VSP-; $n=16 ; p=0.42$ ). b CSF MMP-9 levels during post-SAH days $0-14$ in SAH patients showed no association with presence (VSP $+; n=17)$ or absence of angiographic vasospasm (VSP-; $n=15 ; p=0.32$ )

cerebral ischemia after SAH [25]. Furthermore, there is growing evidence supporting the important role of initial global ischemia in brain injury following SAH [25]. MMPs are known to play important and different roles in acute ischemic brain injuries as well as in brain recovery from such injuries. Elevation of MMPs is associated with worse clinical outcome in acute peri-stroke phase while subacute MMP elevation promotes remodeling and recovery following ischemic injury [26]. In concordance with these studies, we showed for the first time that early elevation of MMP-9 in CSF is associated with poor 3-month clinical outcome in human SAH subjects. Although we are unable to establish the source of CSF MMP-9 in this clinical study, our data suggest that MMP-9 likely plays an important role in early brain injury following SAH.

We also found a positive correlation between blood and CSF MMP-9 levels early in SAH course. Given their relative concentrations, it is conceivable that this positive correlation may represent blood MMP-9 leaking across the BBB into CSF. Whether blood and CSF MMP-9 play a direct pathogenic role in early brain injury or act as a surrogate for poor outcome mediated by degree of BBB disruption will need to be determined by more mechanistic in vitro studies. In our data, while MMP-9 in blood and CSF are associated with poor SAH outcome, they are not associated with angiographic vasospasm, suggesting that MMP-9 may lead to brain injury independent of vasospasm. These results support the growing hypothesis that there may be divergent pathophysiologic pathways leading to angiographic vasospasm and poor outcome in SAH [27, 28].

We also demonstrated, for the first time in humans, that elevation of peripheral neutrophil count is associated with both vasospasm and poor outcome in SAH. Prior clinical studies have established that blood leukocyte elevation at single time points are associated with cerebral vasospasm $[9,14]$, which we replicated in our study. However, none of these studies specifically examined neutrophils. Data from our study support results from animal studies demonstrating that reduced neutrophil activity is associated with less brain injury and better outcome in SAH [29, 30]. The correlation between early neutrophil elevation and blood MMP-9 elevation raise the possibility that neutrophils may mediate poor SAH outcome partly via MMP-9 release.

Our study has several caveats, the most important one being that this is an observational association study. While we carefully studied a prospective and well-phenotyped SAH cohort with longitudinal temporal data and measured MMP-9 in CSF as well as in blood, the overall sample size of this study is modest. With a small sample size, we have no power to meaningfully adjust for all confounders or to perform subset analysis. Given the modest sample size, we limited the risk of multiple comparisons by focusing on a single molecular marker - MMP-9, a major product of neutrophils, in two compartments (blood and ventricular CSF). However, we recognize that SAH-related early brain injury is likely associated with changes in multiple interacting molecular pathways. Our data support MMP-9 as a potentially important mediator of poor SAH outcome, but we cannot comment on the specific pathophysiologic mechanism connecting MMP-9 and brain injury. For example, other important MMPs, in particular, the other gelatinase - MMP-2, is also known to mediate important neuroinflammatory responses [31, 32] and chronic vascular inflammatory processes such as vascular cognitive impairment [33]. In this exploratory study, we chose to focus on the acute inflammatory cascade involving the neutrophil - MMP-9 axis - because neutrophils are major sources of MMP-9 but not of MMP-2 [34]. We acknowledge that MMP-2 may very well play a role in SAH-related cerebral injury and will examine MMP-2 in the future. 
Clinically, due to patient safety considerations, we were only able to sample SAH subjects' blood at four time points between post-SAH days $0-14$, and sample CSF only from EVD systems. This limits our ability to measure and correlate blood and CSF leukocyte and neutrophil counts reliably to examine the time course and degree of inflammation within the CSF compartment and its contribution to MMP-9 release in SAH. Moreover, we can only collect CSF samples from subjects who had EVD placement and CSF drainage for a clinical indication due to ethical considerations. This subset of subjects with both CSF and blood samples available were chosen for MMP-9 analysis. This may bias the selection of subjects for CSF biomarker study to those with clinical symptoms of hydrocephalus and those with higher $\mathrm{HH}$ grade, and thereby limit the generalizability of our results. In summary, while results from this initial study suggest that early peripheral inflammatory response and MMP-9 may be an important predictor of SAH clinical outcome, larger prospective studies, studies of other molecular mediators of SAH outcome, and in-depth bench studies are needed to understand and validate these findings.

Acknowledgment The authors thank Drs. F.A. Sorond and G.V. Henderson (Deparment of Neurology, Brigham and Women's Hospital) for subject recruitment, Dr. A.H. Ropper (Deparment of Neurology, Brigham and Women's Hospital) for advice and funding support, and the Harvard Clinical Translational Science Center for supporting study infrastructure.

This study was sponsored by the American Heart Association (10CRP2610341, Chou), Harvard CTSC/NIH (5KL2RR025757, Chou), NINDS (K23-NS073806, Chou; R21-NS52498, Ning; R01NS48422, Ning; R37-NS37074, Lo; P01-NS55104, Lo), and a departmental research discretionary fund (Chou).

\section{References}

1. Johnston SC, Selvin S, Gress DR. The burden, trends, and demographics of mortality from subarachnoid hemorrhage. Neurology. 1998;50(5):1413-8.

2. Cahill J, Calvert JW, Zhang JH. Mechanisms of early brain injury after subarachnoid hemorrhage. J Cereb Blood Flow Metab. 2006;26(11):1341-53. doi:10.1038/sj.jcbfm.9600283.

3. Kassell NF, Torner JC, Haley Jr EC, Jane JA, Adams HP, Kongable GL. The International Cooperative Study on the timing of aneurysm surgery. Part 1: Overall management results. J Neurosurg. 1990;73(1):18-36.

4. Pluta RM, Hansen-Schwartz J, Dreier J, Vajkoczy P, Macdonald RL, Nishizawa $S$, et al. Cerebral vasospasm following subarachnoid hemorrhage: time for a new world of thought. Neurol Res. 2009;31(2):151-8.

5. Sercombe R, Dinh YR, Gomis P. Cerebrovascular inflammation following subarachnoid hemorrhage. Jpn J Pharmacol. 2002;88 (3):227-49.

6. Sozen T, Tsuchiyama R, Hasegawa Y, Suzuki H, Jadhav V, Nishizawa $\mathrm{S}$, et al. Immunological response in early brain injury after SAH. Acta Neurochir 110(Pt 1):57-61. doi:10.1007/978-37091-0353-1_10.
7. Fassbender K, Hodapp B, Rossol S, Bertsch T, Schmeck J, Schutt $\mathrm{S}$, et al. Inflammatory cytokines in subarachnoid haemorrhage: association with abnormal blood flow velocities in basal cerebral arteries. J Neurol Neurosurg Psychiatry. 2001;70 (4):534-7.

8. Dumont AS, Dumont RJ, Chow MM, Lin CL, Calisaneller T, Ley $\mathrm{KF}$, et al. Cerebral vasospasm after subarachnoid hemorrhage: putative role of inflammation. Neurosurgery. 2003;53(1):123-33. discussion 33-5.

9. McGirt MJ, Mavropoulos JC, McGirt LY, Alexander MJ, Friedman $\mathrm{AH}$, Laskowitz DT, et al. Leukocytosis as an independent risk factor for cerebral vasospasm following aneurysmal subarachnoid hemorrhage. J Neurosurg. 2003;98(6):1222-6.

10. Niikawa S, Hara S, Ohe N, Miwa Y, Ohkuma A. Correlation between blood parameters and symptomatic vasospasm in subarachnoid hemorrhage patients. Neurol Medico-chirur. 1997;37((12):881-4. discussion 4-5.

11. Spallone A, Acqui M, Pastore FS, Guidetti B. Relationship between leukocytosis and ischemic complications following aneurysmal subarachnoid hemorrhage. Surg Neurol. 1987;27 (3):253-8.

12. Dhar R, Diringer MN. The burden of the systemic inflammatory response predicts vasospasm and outcome after subarachnoid hemorrhage. Neurocrit care. 2008;8(3):404-12.

13. Yoshimoto Y, Tanaka Y, Hoya K. Acute systemic inflammatory response syndrome in subarachnoid hemorrhage. Stroke. 2001;32 (9):1989-93.

14. Weir B, Disney L, Grace M, Roberts P. Daily trends in white blood cell count and temperature after subarachnoid hemorrhage from aneurysm. Neurosurgery. 1989;25(2):161-5.

15. Maiuri F, Gallicchio B, Donati P, Carandente M. The blood leukocyte count and its prognostic significance in subarachnoid hemorrhage. J Neurosurg Sci. 1987;31(2):45-8.

16. Cuzner ML, Opdenakker G. Plasminogen activators and matrix metalloproteases, mediators of extracellular proteolysis in inflammatory demyelination of the central nervous system. J Neuroimmunol. 1999;94(1-2):1-14.

17. Guo ZD, Sun XC, Zhang JH. Mechanisms of early brain injury after SAH: matrix metalloproteinase 9. Acta Neurochirur.110(Pt 1):63-5. doi:10.1007/978-3-7091-0353-1_11.

18. Gu Z, Kaul M, Yan B, Kridel SJ, Cui J, Strongin A, et al. Snitrosylation of matrix metalloproteinases: signaling pathway to neuronal cell death. Science. 2002;297(5584):1186-90. doi:10.1126/science.1073634297/5584/1186.

19. Lo EH, Wang X, Cuzner ML. Extracellular proteolysis in brain injury and inflammation: role for plasminogen activators and matrix metalloproteinases. J Neurosci Res. 2002;69(1):19 .

20. Watts SW, Thakali K, Smark C, Rondelli C, Fink GD. Big ET-1 processing into vasoactive peptides in arteries and veins. Vasc Pharmacol. 2007;47(5-6):302-12.

21. Fernandez-Patron C, Zouki C, Whittal R, Chan JS, Davidge ST, Filep JG. Matrix metalloproteinases regulate neutrophil-endothelial cell adhesion through generation of endothelin-1[1-32]. FASEB J. 2001;15(12):2230-40.

22. Fernandez-Patron C, Radomski MW, Davidge ST. Vascular matrix metalloproteinase-2 cleaves big endothelin-1 yielding a novel vasoconstrictor. Circ Res. 1999;85(10):906-11.

23. Wang X, Mori T, Jung JC, Fini ME, Lo EH. Secretion of matrix metalloproteinase-2 and -9 after mechanical trauma injury in rat cortical cultures and involvement of MAP kinase. J Neurotrauma. 2002;19(5):615-25.

24. Chou SH-Y, Lee P-S, Konigsberg RG, Gallacci D, Chiou T, Arai $\mathrm{K}$, et al. Plasma-type gelsolin is decreased in human blood and cerebrospinal fluid following subarachnoid hemorrhage. Stroke. 2011 (in press). 
25. Ostrowski RP, Colohan AR, Zhang JH. Molecular mechanisms of early brain injury after subarachnoid hemorrhage. Neurol Res. 2006;28(4):399-414.

26. Zhao BQ, Wang S, Kim HY, Storrie H, Rosen BR, Mooney DJ, et al. Role of matrix metalloproteinases in delayed cortical responses after stroke. Nat Med. 2006;12(4):441-5.

27. Macdonald RL, Pluta RM, Zhang JH. Cerebral vasospasm after subarachnoid hemorrhage: the emerging revolution. Nat Clin Pract. 2007;3(5):256-63.

28. Zemke D, Farooq MU, Mohammed Yahia A, Majid A. Delayed ischemia after subarachnoid hemorrhage: result of vasospasm alone or a broader vasculopathy? Vasc Med. 2007;12(3):243-9. doi:10.1177/1358863X07081316.

29. Friedrich V, Flores R, Muller A, Bi W, Peerschke EI, Sehba FA. Reduction of neutrophil activity decreases early microvascular injury after subarachnoid haemorrhage. J Neuroinflam. 8(1):103. doi:10.1186/1742-2094-8-103.
30. Provencio JJ, Fu X, Siu A, Rasmussen PA, Hazen SL, Ransohoff RM. CSF neutrophils are implicated in the development of vasospasm in subarachnoid hemorrhage. Neurocrit Care. 2010;12(2):244-51.

31. Rosenberg GA. Matrix metalloproteinases in neuroinflammation. Glia. 2002;39(3):279-91. doi:10.1002/glia.10108.

32. Opdenakker G, Van den Steen PE, Dubois B, Nelissen I, Van Coillie E, Masure S, et al. Gelatinase B functions as regulator and effector in leukocyte biology. J Leukoc Biol. 2001;69(6):851-9.

33. Candelario-Jalil E, Thompson J, Taheri S, Grossetete M, Adair $\mathrm{JC}$, Edmonds E, et al. Matrix metalloproteinases are associated with increased blood-brain barrier opening in vascular cognitive impairment. Stroke. 2011;42(5):1345-50. doi:10.1161/ STROKEAHA.110.600825.

34. Ardi VC, Kupriyanova TA, Deryugina EI, Quigley JP. Human neutrophils uniquely release TIMP-free MMP-9 to provide a potent catalytic stimulator of angiogenesis. Proc Natl Acad Sci U S A. 2007;104(51):20262-7. doi:10.1073/pnas.0706438104. 